\title{
Weakly-Supervised Neural Text Classification
}

\author{
Yu Meng, Jiaming Shen, Chao Zhang, Jiawei Han \\ Department of Computer Science, University of at Illinois Urbana-Champaign, IL, USA \\ \{yumeng5, js2, czhang82, hanj\}@illinois.edu
}

\begin{abstract}
Deep neural networks are gaining increasing popularity for the classic text classification task, due to their strong expressive power and less requirement for feature engineering. Despite such attractiveness, neural text classification models suffer from the lack of training data in many real-world applications. Although many semisupervised and weakly-supervised text classification models exist, they cannot be easily applied to deep neural models and meanwhile support limited supervision types. In this paper, we propose a weakly-supervised method that addresses the lack of training data in neural text classification. Our method consists of two modules: (1) a pseudo-document generator that leverages seed information to generate pseudo-labeled documents for model pre-training, and (2) a self-training module that bootstraps on real unlabeled data for model refinement. Our method has the flexibility to handle different types of weak supervision and can be easily integrated into existing deep neural models for text classification. We have performed extensive experiments on three real-world datasets from different domains. The results demonstrate that our proposed method achieves inspiring performance without requiring excessive training data and outperforms baseline methods significantly.
\end{abstract}

\section{CCS CONCEPTS}

- Information systems $\rightarrow$ Clustering and classification; • Computing methodologies $\rightarrow$ Learning paradigms; Neural networks;

\section{KEYWORDS}

Text Classification; Weakly-supervised Learning; Pseudo Document Generation; Neural Classification Model

\section{ACM Reference Format:}

Yu Meng, Jiaming Shen, Chao Zhang, Jiawei Han. 2018. Weakly-Supervised Neural Text Classification. In The 27th ACM International Conference on Information and Knowledge Management (CIKM '18), October 22-26, 2018, Torino, Italy. ACM, New York, NY, USA, 10 pages. https://doi.org/10.1145/ 3269206.3271737

\section{INTRODUCTION}

Text classification plays a fundamental role in a wide variety of applications, ranging from sentiment analysis [27] to document categorization [32] and query intent classification [29]. Recently, deep neural models - including convolutional neural networks

Permission to make digital or hard copies of all or part of this work for personal or classroom use is granted without fee provided that copies are not made or distributed for profit or commercial advantage and that copies bear this notice and the full citation on the first page. Copyrights for components of this work owned by others than ACM must be honored. Abstracting with credit is permitted. To copy otherwise, or republish, to post on servers or to redistribute to lists, requires prior specific permission and/or a fee. Request permissions from permissions@acm.org.

CIKM '18, October 22-26, 2018, Torino, Italy

(C) 2018 Association for Computing Machinery.

ACM ISBN 978-1-4503-6014-2/18/10 . \$ \$15.00

https://doi.org/10.1145/3269206.3271737
(CNNs) $[11,12,35,36]$ and recurrent neural networks (RNNs) [22, $23,32]-$ have demonstrated superiority for this classic task. The attractiveness of these neural models for text classification is mainly two-fold. First, they can largely reduce feature engineering efforts by automatically learning distributed representations that capture text semantics. Second, they enjoy strong expressive power and thus have the potential to better learn from the data and yield better classification performance.

Despite the attractiveness and increasing popularity of neural models for text classification, the lack of training data is still a key bottleneck that prohibits them from being adopted in many practical scenarios. Indeed, training a deep neural model for text classification can easily consume million-scale labeled documents. Collecting such training data requires domain experts to read through millions of documents and carefully label them with domain knowledge, which is often too expensive to realize.

To address the label scarcity bottleneck, we study the problem of learning neural models for text classification under weak supervision. In many scenarios, while users cannot afford to label many documents for training neural models, they can provide a small amount of seed information for the classification task. Such seed information may arrive in various forms: either a set of representative keywords for each class, or a few (less than a dozen) labeled documents, or even only the surface names of the classes. Such a problem is called weakly-supervised text classification.

There have been many studies related to weakly-supervised text classification. However, training neural models for text classification under weak supervision remains an open research problem. Several semi-supervised neural models have been proposed [18, 31], but they still require hundreds or even thousands of labeled training examples, which are not available in the weakly supervised setting [20]. Along another line, there are existing methods that perform weakly-supervised text classification, including latent variable models [14] and embedding-based methods [15, 28]. These models have the following limitations: (1) supervision inflexibility: they can only handle one type of seed information, either a collection of labeled documents or a set of class-related keywords, which restricts their applicabilities; (2) seed sensitivity: the "seed supervision" from users completely controls the model training process, making the learned model very sensitive to the initial seed information; (3) limited extensibility: these methods are specific to either latent variable models or embedding methods, and cannot be readily applied to learn deep neural models based on CNN or RNN.

We propose a new method, named WeSTClass, for WeaklySupervised Text Classification. As shown in Figure 1, WESTCLAss contains two modules to address the above challenges. The first module is a pseudo-document generator, which leverages seed information to generate pseudo documents as synthesized training data. By assuming word and document representations reside in the same semantic space, we generate pseudo documents for each 


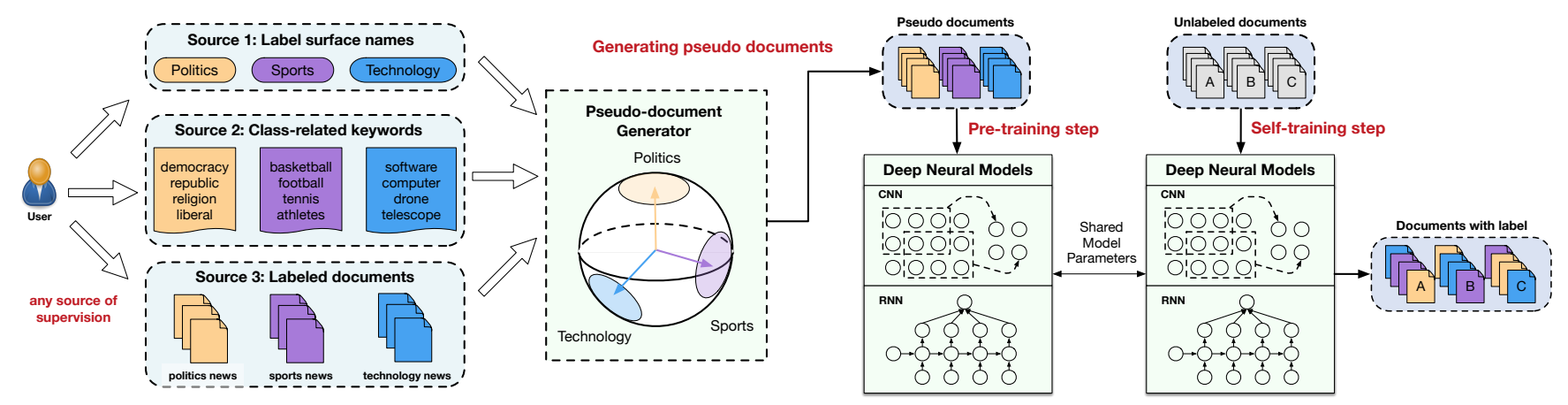

Figure 1: Our proposed weakly-supervised neural text classification model consists of two key modules: (1) a pseudo-document generator that leverages seed information to generate pseudo-labeled documents for model pre-training, and (2) a self-training module that bootstraps on real unlabeled data for model refinement.

class by modeling the semantics of each class as a high-dimensional spherical distribution [7], and further sampling keywords to form pseudo documents. The pseudo document generator can not only expand user-given seed information for better generalization, but also handle different types of seed information (e.g., label surface names, class-related keywords, or a few labeled documents) flexibly.

The second key module of our method is a self-training module that fits real unlabeled documents for model refinement. First, the self-training module uses pseudo documents to pre-train either CNN-based or RNN-based models to produce an initial model, which serves as a starting point in the subsequent model refining process. Then, it applies a self-training procedure, which iteratively makes predictions on real unlabeled documents and leverages highconfidence predictions to refine the neural model.

In summary, this paper makes the following contributions:

(1) We design WeSTCLAss method for addressing the label scarcity bottleneck of neural text classification. To the best of our knowledge, WESTCLASS is the first weakly-supervised text classification method that can be applied to most existing neural models and meanwhile handle different types of seed information.

(2) We propose a novel pseudo document generator by modeling the class semantic as a spherical distribution. The generator is able to generate pseudo documents that are highly correlated to each class, and meanwhile effectively expands user-provided seed information for better generalization.

(3) We propose a self-training algorithm for training deep neural models by leveraging pseudo documents. The self-training algorithm can iteratively bootstrap the unlabeled data to obtain high-quality deep neural models, and is generic enough to be integrated into either $\mathrm{CNN}$-based or RNN-based models.

(4) We conduct a thorough evaluation of our proposed method on three real-world datasets from different domains. The experiment results show that our method can achieve inspiring text classification performance even without excessive training data and outperforms various baselines.

\section{RELATED WORK}

In this section, we review existing studies for weakly-supervised text classification, which can be categorized into two classes: (1) latent variable models; and (2) embedding-based models.

\subsection{Latent Variable Models}

Existing latent variable models for weakly-supervised text classification mainly extend topic models by incorporating user-provided seed information. Specifically, semi-supervised PLSA [16] extends the classic PLSA model by incorporating a conjugate prior based on expert review segments (topic keywords or phrases) to force extracted topics to be aligned with provided review segments. [9] encodes prior knowledge and indirect supervision in constraints on posteriors of latent variable probabilistic models. Descriptive LDA [6] uses an LDA model as the describing device to infer Dirichlet priors from given category labels and descriptions. The Dirichlet priors guides LDA to induce the category-aware topics. Seed-guided topic model [14] takes a small set of seed words that are relevant to the semantic meaning of the category, and then predicts the category labels of the documents through two kinds of topic influence: category-topics and general-topics. The labels of the documents are inferred based on posterior category-topic assignment. Our method differs from these latent variable models in that it is a weaklysupervised neural model. As such, it enjoys two advantages over these latent variable models: (1) it has more flexibility to handle different types of seed information which can be a collection of labeled documents or a set of seed keywords related to each class; (2) it does not need to impose assumptions on document-topic or topic-keyword distributions, but instead directly uses massive data to learn distributed representations to capture text semantics.

\section{$2.2 \quad$ Embedding-based Models}

Embedding-based weakly supervised models use seed information to derive vectorized representations for documents and label names for the text classification task. Dataless classification [5, 24] takes category names and projects each word and document into the same semantic space of Wikipedia concepts. Each category is represented with words in the category label. The document classification is performed based on the vector similarity between a document and a category using explicit semantic analysis [8]. Unsupervised neural categorization [15] takes category names as input and applies a cascade embedding approach: First the seeded category names and other significant phrases (concepts) are embedded into vectors for capturing concept semantics. Then the concepts are embedded into 
a hidden category space to make the category information explicit. Predictive text embedding [28] is a semi-supervised algorithm that utilizes both labeled and unlabeled documents to learn text embedding specifically for a task. Labeled data and different levels of word co-occurrence information are first represented as a largescale heterogeneous text network and then embedded into a low dimensional space that preserves the semantic similarity of words and documents. Classification is performed by using one-vs-rest logistic regression model as classifier and the learned embedding as input. Compared with our method, these embedding-based weakly supervised methods cannot be directly applied to deep neural models $(\mathrm{CNN}, \mathrm{RNN})$ for the text classification task. Furthermore, while they allow the seed information to directly control the model training process, we introduce a pseudo document generation paradigm which is generalized from the seed information. Hence, our model is less prone to seed information overfitting and enjoys better generalization ability.

\section{PRELIMINARIES}

In this section, we formulate the problem of weakly-supervised text classification, and give an overview of our proposed method.

\subsection{Problem Formulation}

Given a text collection $\mathcal{D}=\left\{D_{1}, \ldots, D_{n}\right\}$ and $m$ target classes $C=\left\{C_{1}, \ldots, C_{m}\right\}$, text classification aims to assign a class label $C_{j} \in C$ to each document $D_{i} \in \mathcal{D}$. To characterize each class, traditional supervised text classification methods rely on large amounts of labeled documents. In this work, we focus on the text classification under weakly-supervised setting where the supervision signal comes from one of the following sources: (1) label surface names: $\mathcal{L}=\left.\left\{L_{j}\right\}\right|_{j=1} ^{m}$, where $L_{j}$ is the surface name for class $C_{j},(2)$ class-related keywords: $\mathcal{S}=\left.\left\{S_{j}\right\}\right|_{j=1} ^{m}$, where $S_{j}=\left\{w_{j, 1}, \ldots, w_{j, k}\right\}$ represents a set of $k$ keywords in class $C_{j}$, and (3) labeled documents: $\mathcal{D}^{L}=\left.\left\{\mathcal{D}_{j}^{L}\right\}\right|_{j=1} ^{m}$, where $\mathcal{D}_{j}^{L}=\left\{D_{j, 1}, \ldots, D_{j, l}\right\}$ denotes a set of $l$ $(l \ll n)$ labeled documents in class $C_{j}$. In many scenarios, the above weak supervision signals can be easily obtained from users. Finally, we define our problem as follows:

Definition 3.1 (Problem Formulation). Given a text collection $\mathcal{D}=$ $\left\{D_{1}, \ldots, D_{n}\right\}$, target classes $C=\left\{C_{1}, \ldots, C_{m}\right\}$, and weak supervision from either $\mathcal{L}, \mathcal{S}$ or $\mathcal{D}^{L}$, the weakly-supervised text classification task aims to assign a label $C_{j} \in C$ to each $D_{i} \in \mathcal{D}$.

\subsection{Method Overview}

Our proposed weakly-supervised text classification method contains two key modules. The first one is a pseudo-document generator that unifies seed information and outputs pseudo documents for model training. We assume words and documents share a joint semantic space which provides flexibility for handling different types of seed information. Then, we model each class as a highdimensional spherical distribution from which keywords are sampled to form pseudo documents as training data. The second key module of our method is a self-training module that can be easily integrated into existing deep neural models, either CNN-based or RNN-based. It first uses the generated pseudo documents to pre-train neural models, which allows the model to start with a good initialization. Then, a self-training procedure is applied to iteratively refine the neural model using unlabeled real documents based on the model's high-confidence predictions. We show the entire process of our method in Figure 1.

\section{PSEUDO DOCUMENT GENERATION}

In this section, we describe the details of the pseudo-document generator, which leverages seed information to generate a bunch of pseudo documents that are correlated to each class. Below, we first introduce how to model class distributions in a joint semantic space with words and documents, and then describe the pseudo document generation process.

\subsection{Modeling Class Distribution}

To effectively leverage user-provided seed information and capture the semantic correlations between words, documents and classes, we assume words and documents share a joint semantic space, based on which we learn a generative model for each class to generate pseudo documents.

Specifically, we first use the Skip-Gram model [17] to learn $p$ dimensional vector representations of all the words in the corpus. Furthermore, since directional similarities between vectors are more effective in capturing semantic correlations [2,13, 25], we normalize all the $p$-dimensional word embeddings so that they reside on a unit sphere in $\mathbb{R}^{p}$, which is the joint semantic space. We call it "joint" because we assume pseudo document vectors reside on the same unit sphere as well, which we will explain in Section 4.2. We retrieve a set of keywords in the semantic space that are correlated to each class based on the seed information. We describe how to handle different types of seed information as follows:

- Label surface names: When only label surface names $\mathcal{L}$ are given as seed information, for each class $j$ we use the embedding of its surface name $L_{j}$ to retrieve top- $t$ nearest words in the semantic space. We set $t$ to be the largest number that does not results in shared words across different classes.

- Class-related keywords: When users provide a list of related keywords $S_{j}$ for each class $j$, we use the embeddings of these seed keywords to find top- $t$ keywords in the semantic space, by measuring the average similarity to the seed keywords.

- Labeled documents: When users provide a small number of documents $\mathcal{D}_{j}^{L}$ that are correlated with class $j$, we first extract $t$ representative keywords in $\mathcal{D}_{j}^{L}$ using tf-idf weighting, and then consider them as class-related keywords.

After obtaining a set of keywords that are correlated with each class, we model the semantic of each class as a von Mises Fisher (vMF) distribution $[2,10]$, which models word embeddings on a unit sphere in $\mathbb{R}^{p}$ and has been shown effective for various tasks $[3,34]$. Specifically, we define the probability distribution of a class as:

$$
f(\boldsymbol{x} ; \boldsymbol{\mu}, \kappa)=c_{p}(\kappa) e^{\kappa \boldsymbol{\mu}^{T} \boldsymbol{x}},
$$

where $\kappa \geq 0,\|\boldsymbol{\mu}\|=1, p \geq 2$ and the normalization constant $c_{p}(\kappa)$ is given by

$$
c_{p}(\kappa)=\frac{\kappa^{p / 2-1}}{(2 \pi)^{p / 2} I_{p / 2-1}(\kappa)},
$$


where $I_{r}(\cdot)$ represents the modified Bessel function of the first kind at order $r$. We justify our choice of the vMF distribution as follows: the vMF distribution has two parameters-the mean direction $\boldsymbol{\mu}$ and the concentration parameter $\kappa$. The distribution of keywords on the unit sphere for a specific class concentrates around the mean direction $\boldsymbol{\mu}$, and is more concentrated if $\kappa$ is large. Intuitively, the mean direction $\boldsymbol{\mu}$ acts as a semantic focus on the unit sphere, and produces relevant semantic embeddings around it, where concentration degree is controlled by the parameter $\kappa$.

Now that we have leveraged the seed information to obtain a set of keywords for each class on the unit sphere, we can use these correlated keywords to fit a vMF distribution $f(\boldsymbol{x} ; \boldsymbol{\mu}, \kappa)$. Specifically, let $X$ be a set of vectors for the keywords on the unit sphere, i.e.,

$$
X=\left\{\boldsymbol{x}_{i} \in \mathbb{R}^{p} \mid \boldsymbol{x}_{i} \text { drawn from } f(\boldsymbol{x} ; \boldsymbol{\mu}, \kappa), 1 \leq i \leq t\right\},
$$

then we use the maximum likelihood estimates [2,26] for finding the parameters $\hat{\mu}$ and $\hat{\kappa}$ of the vMF distribution:

$$
\hat{\mu}=\frac{\sum_{i=1}^{t} x_{i}}{\left\|\sum_{i=1}^{t} x_{i}\right\|}
$$

and

$$
\frac{I_{p / 2}(\hat{\kappa})}{I_{p / 2-1}(\hat{\kappa})}=\frac{\left\|\sum_{i=1}^{t} x_{i}\right\|}{t} .
$$

Obtaining an analytic solution for $\hat{\kappa}$ is infeasible because the formula involves an implicit equation which is a ratio of Bessel functions. We thus use a numerical procedure based on Newton's method [2] to derive an approximation of $\hat{\kappa}$.

\subsection{Generating Pseudo Documents}

To generate a pseudo document $D_{i}^{*}$ (we use $D_{i}^{*}$ instead of $D_{i}$ to denote it is a pseudo document) of class $j$, we propose a generative mixture model based on class $j$ 's distribution $f\left(\boldsymbol{x} ; \boldsymbol{\mu}_{j}, \kappa_{j}\right)$. The mixture model repeatedly generates a number of terms to form a pseudo document; when generating each term, the model chooses from a background distribution with probability $\alpha(0<\alpha<1)$ and from the class-specific distribution with probability $1-\alpha$.

The class-specific distribution is defined based on class $j$ 's distribution $f\left(\boldsymbol{x} ; \boldsymbol{\mu}_{j}, \kappa_{j}\right)$. Particularly, we first sample a document vector $\boldsymbol{d}_{i}$ from $f\left(\boldsymbol{x} ; \boldsymbol{\mu}_{j}, \kappa_{j}\right)$, then build a keyword vocabulary $V_{d_{i}}$ for $\boldsymbol{d}_{i}$ that contains the top- $\gamma$ words with most similar word embedding with $\boldsymbol{d}_{i}$. These $\gamma$ words in $V_{d_{i}}$ are highly semantically relevant with the topic of pseudo document $D_{i}^{*}$ and will appear frequently in $D_{i}^{*}$. Each term of a pseudo document is generated according to the following probability distribution:

$$
p\left(w \mid \boldsymbol{d}_{i}\right)= \begin{cases}\alpha p_{B}(w) & w \notin V_{d_{i}} \\ \alpha p_{B}(w)+(1-\alpha) \frac{\exp \left(\boldsymbol{d}_{i}^{T} \boldsymbol{v}_{w}\right)}{\sum_{w^{\prime} \in V_{d_{i}}} \exp \left(\boldsymbol{d}_{i}^{T} \boldsymbol{v}_{w^{\prime}}\right)} & w \in V_{d_{i}}\end{cases}
$$

where $\boldsymbol{v}_{w}$ is the word embedding for $w$ and $p_{B}(w)$ is the background distribution for the entire corpus.

Note that we generate document vectors from $f\left(\boldsymbol{x} ; \boldsymbol{\mu}_{j}, \kappa_{j}\right)$ instead of fixing them to be $\boldsymbol{\mu}_{j}$. The reason is that some class (e.g., Sports) may cover a wide range of topics (e.g., athlete activities, sport competitions, etc.), but using $\boldsymbol{\mu}_{j}$ as the pseudo document vector will only attract words that are semantically similar to the centroid direction of a class. Sampling pseudo document vectors

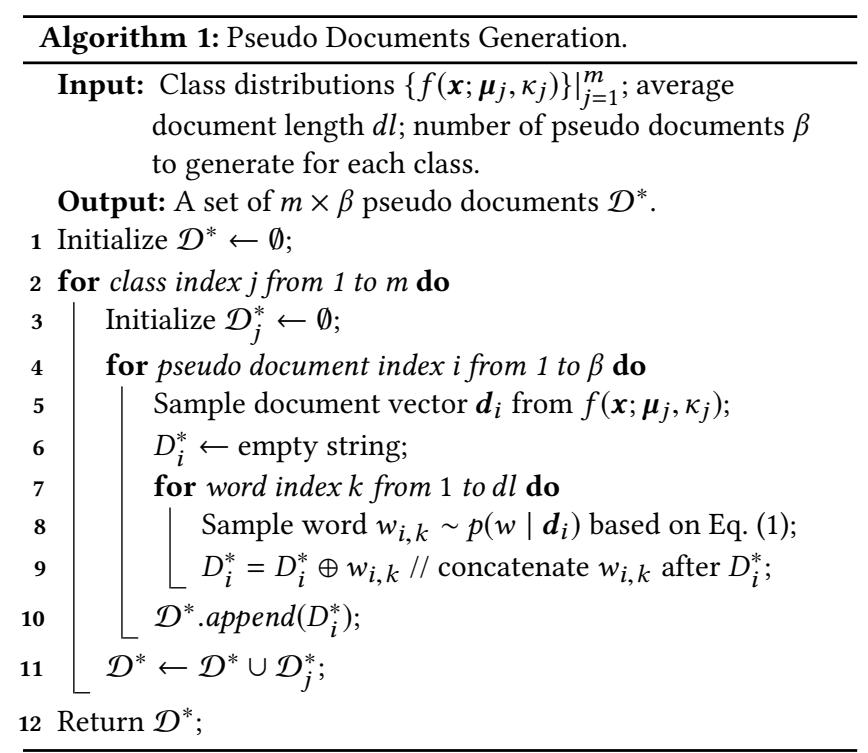

from the distribution, however, allows the generated pseudo documents to be more semantically diversified and thus cover more information about the class. Consequently, models trained on such more diversified pseudo documents are expected to have better generalization ability.

Algorithm 1 shows the whole process of generating a collection of $\beta$ pseudo documents per class. For each class $j$, given the learned class distributions and the average length of pseudo documents $d l^{1}$, we draw a document vector $\boldsymbol{d}_{i}$ from class $j$ 's distribution $f\left(\boldsymbol{x} ; \boldsymbol{\mu}_{j}, \kappa_{j}\right)$. After that, we generate $d l$ words sequentially based on $\boldsymbol{d}_{i}$ and add the generated document into the pseudo document collection $\mathcal{D}_{j}^{*}$ of class $j$. After the above process repeats $\beta$ times, we finally obtain $\mathcal{D}_{j}^{*}$ which contains $\beta$ pseudo documents for class $j$.

\section{NEURAL MODELS WITH SELF-TRAINING}

In this section, we present the self-training module that trains deep neural models with the generated pseudo documents. The self-training module first uses the pseudo documents to pre-train a deep neural network, and then iteratively refines the trained model on the real unlabeled documents in a bootstrapping fashion. In the following, we first present the pre-training and the selftraining steps in Section 5.1 and 5.2, and then demonstrate how the framework can be instantiated with $\mathrm{CNN}$ and RNN models in Section 5.3.

\subsection{Neural Model Pre-training}

As we have obtained pseudo documents for each class, we use them to pre-train a neural network $M^{2}$. A naive way of creating the label for a pseudo document $D_{i}^{*}$ is to directly use the associated class label that $D_{i}^{*}$ is generated from, i.e. using one-hot encoding where the generating class takes value 1 and all other classes are set to 0 . However, this naive strategy often causes the neural model to

\footnotetext{
${ }^{1}$ The length of each pseudo document can be either manually set or equal to the average document length in the real document collection.

${ }^{2}$ When the supervision source is labeled documents, these seed documents will be used to augment the pseudo document set during the pre-training step.
} 
overfit to the pseudo documents and have limited performance when classifying real documents, due to the fact that the generated pseudo documents do not contain word ordering information. To tackle this problem, we create pseudo labels for pseudo documents. In Equation (1), we design pseudo documents to be generated from a mixture of background and class-specific word distributions, controlled by a balancing parameter $\alpha$. Such a process naturally leads to our design of the following procedure for pseudo label creation: we evenly split the fraction of the background distribution into all $m$ classes, and set the pseudo label $\boldsymbol{l}_{i}$ for pseudo document $D_{i}^{*}$ as

$$
l_{i j}= \begin{cases}(1-\alpha)+\alpha / m & D_{i}^{*} \text { is generated from class } j \\ \alpha / m & \text { otherwise }\end{cases}
$$

After creating the pseudo labels, we pre-train a neural model $M$ by generating $\beta$ pseudo documents for each class, and minimizing the KL divergence loss from the neural network outputs $Y$ to the pseudo labels $L$, namely

$$
\text { loss }=K L(L \| Y)=\sum_{i} \sum_{j} l_{i j} \log \frac{l_{i j}}{y_{i j}}
$$

We will detail how we instantiate the neural model $M$ shortly in Section 5.3

\subsection{Neural Model Self-training}

While the pre-training step produces an initial neural model $M$, the performance of the $M$ is not the best one can hope for. The major reason is that the pre-trained model $M$ only uses the set of pseudo documents but fails to take advantage of the information encoded in the real unlabeled documents. The self-training step is designed to tackle the above issues. Self-training $[19,21]$ is a common strategy used in classic semi-supervised learning scenarios. The rationale behind self-training is to first train the model with labeled data, and then bootstrap the learning model with its current highly-confident predictions.

After the pre-training step, we use the pre-trained model to classify all unlabeled documents in the corpus and then apply a self-training strategy to improve the current predictions. During self-training, we iteratively compute pseudo labels based on current predictions and refine model parameters by training the neural network with pseudo labels. Given the current outputs $Y$, the pseudo labels are computed using the same self-training formula as in [30]:

$$
l_{i j}=\frac{y_{i j}^{2} / f_{j}}{\sum_{j^{\prime}} y_{i j^{\prime}}^{2} / f_{j^{\prime}}}
$$

where $f_{j}=\sum_{i} y_{i j}$ is the soft frequency for class $j$.

Self-training is performed by iteratively computing pseudo labels and minimizing the KL divergence loss from the current predictions $Y$ to the pseudo labels $L$. This process terminates when less than $\delta \%$ of the documents in the corpus have class assignment changes.

Although both pre-training and self-training create pseudo labels and use them to train neural models, it is worth mentioning the difference between them: in pre-training, pseudo labels are paired with generated pseudo documents to distinguish them from given labeled documents (if provided) and prevent the neural models from overfitting to pseudo documents; in self-training, pseudo labels are paired with every unlabeled real documents from corpus and reflect current high confidence predictions.

\subsection{Instantiating with $\mathrm{CNNs}$ and RNNs}

As mentioned earlier, our method for text classification is generic enough to be applied to most existing deep neural models. In this section, we instantiate the framework with two mainstream deep neural network models: convolution neural networks $(\mathrm{CNN})$ and recurrent neural networks (RNN), by focusing on how they are used to learn document representations and perform classification.

5.3.1 CNN-Based Models. CNNs have been explored for text classification [12]. When instantiating our framework with $\mathrm{CNN}$, the input to a $\mathrm{CNN}$ is a document of length $d l$ represented by a concatenation of word vectors, i.e.,

$$
d=x_{1} \oplus x_{2} \oplus \cdots \oplus x_{d l},
$$

where $\boldsymbol{x}_{i} \in \mathbb{R}^{p}$ is the $p$ dimensional word vector of the $i$ th word in the document. We use $\boldsymbol{x}_{i: i+j}$ to represent the concatenation of word vectors $\boldsymbol{x}_{i}, \boldsymbol{x}_{i+1}, \ldots, \boldsymbol{x}_{i+j}$. For window size of $h$, a feature $c_{i}$ is generated from a window of words $x_{i: i+h-1}$ by the following convolution operation

$$
c_{i}=f\left(\boldsymbol{w} \cdot \boldsymbol{x}_{i: i+h-1}+b\right),
$$

where $b \in \mathbb{R}$ is a bias term, $w \in \mathbb{R}^{h p}$ is the filter operating on $h$ words. For each possible size- $h$ window of words, a feature map is generated as

$$
c=\left[c_{1}, c_{2}, \ldots, c_{d l-h+1}\right] .
$$

Then a max-over-time pooling operation is performed on $c$ to output the maximum value $\hat{c}=\max (\boldsymbol{c})$ as the feature corresponding to this particular filter. If we use multiple filters, we will obtain multiple features that are passed through a fully connected softmax layer whose output is the probability distribution over labels.

5.3.2 RNN-Based Models. Besides CNNs, we also discuss how to instantiate our framework with RNNs. We choose the Hierarchical Attention Network (HAN) [33] as an exemplar RNN-based model. HAN consists of sequence encoders and attention layers for both words and sentences. In our context, the input document is represented by a sequence of sentences $s_{i}, i \in[1, L]$ and each sentence is represented by a sequence of words $w_{i t}, t \in[1, T]$. At time $t$, the GRU [1] computes the new state as

$$
\boldsymbol{h}_{t}=\left(\mathbf{1}-z_{t}\right) \odot \boldsymbol{h}_{t-1}+z_{t} \odot \tilde{\boldsymbol{h}}_{t},
$$

where the update gate vector

$$
z_{t}=\sigma\left(W_{z} \boldsymbol{x}_{t}+U_{z} \boldsymbol{h}_{t-1}+\boldsymbol{b}_{z}\right)
$$

the candidate state vector

$$
\tilde{\boldsymbol{h}}_{t}=\tanh \left(W_{h} \boldsymbol{x}_{t}+\boldsymbol{r}_{t} \odot\left(U_{h} \boldsymbol{h}_{t-1}\right)+\boldsymbol{b}_{h}\right),
$$

the reset gate vector

$$
\boldsymbol{r}_{t}=\sigma\left(W_{r} \boldsymbol{x}_{t}+U_{r} \boldsymbol{h}_{t-1}+\boldsymbol{b}_{r}\right)
$$

and $\boldsymbol{x}_{t}$ is the sequence vector (word embedding or sentence vector) at time $t$. After encoding words and sentences, we also impose the attention layers to extract important words and sentences with the attention mechanism, and derive their weighted average as document representations. 


\section{EXPERIMENTS}

In this section, we evaluate the empirical performance of our method for weakly supervised text classification.

\subsection{Datasets}

We use three corpora from different domains to evaluate the performance of our proposed method: (1) The New York Times: We crawl 13, 081 news articles using the New York Times API $^{3}$. This corpus covers 5 major news topics; (2) AG's News: We use the same AG's News dataset from [36] and take its training set portion (120, 000 documents evenly distributed into 4 classes) as the corpus for evaluation; (3) Yelp Review: We use the Yelp reviews polarity dataset from [36] and take its testing set portion (38, 000 documents evenly distributed into 2 classes) as the corpus for evaluation. Table 1 provides the details of these datasets.

\subsection{Baselines}

We compare WESTCLASS with a wide range of baseline models, described as follows.

- IR with tf-idf: this method accepts either label surface name or class-related keywords as supervision. We treat the label name or keyword set for each class as a query, and score the relevance of document to this class using the tf-idf model. The class with highest relevance score is assigned to the document.

- Topic Model: this method accepts either label surface name or class-related keywords as supervision. We first train the LDA model [4] on the entire corpus. Given a document, we compute the likelihood of observing label surface names or the average likelihood of observing class-related keywords. The class with maximum likelihood will be assigned to the document.

- Dataless [5, 24]: this method ${ }^{4}$ accepts only label surface name as supervision. It leverages Wikipedia and uses Explicit Semantic Analysis [8] to derive vector representations of both labels and documents. The final document class is assigned based on the vector similarity between labels and documents.

- UNEC [15]: this method takes label surface name as its weak supervision. It categorizes documents by learning the semantics and category attribution of concepts inside the corpus. We use the authors' original implementation of this model.

- PTE [28]: this method ${ }^{5}$ uses labeled documents as supervision. It first utilizes both labeled and unlabeled data to learn text embedding and then applies logistic regression model as classifier for text classification.

- CNN [12]: the original CNN model is a supervised text classification model and we extend it to incorporate all three types of supervision sources. If labeled documents are given, we directly train $\mathrm{CNN}$ model on the given labeled documents and then apply it on all unlabeled documents. If label surface names or class-related keywords are given, we first use the above "IR with tf-idf" or "Topic Modeling" method (depending on which one works better) to label all unlabeled documents. Then, we select $\beta$ labeled documents per class to pre-train CNN.

\footnotetext{
${ }^{3}$ http://developer.nytimes.com/

${ }^{4}$ https://cogcomp.org/page/software_view/Descartes

${ }^{5}$ https://github.com/mnqu/PTE
}

Finally, we apply the same self-training module as described in Section 5 to obtain the final classifier.

- HAN [33]: similar to the above CNN model, we extend the original HAN model ${ }^{6}$ to incorporate all three types of supervision sources.

- NoST-(CNN/HAN): this is a variant of WeSTClAss without the self-training module, i.e., after pre-training $\mathrm{CNN}$ or HAN with pseudo documents, we directly apply it to classify unlabeled documents.

- WeSTCLAss-(CNN/HAN): this is the full version of our proposed framework, with both pseudo-document generator and self-training module enabled.

\subsection{Experiment Settings}

We first describe our parameter settings as follows. For all datasets, we use the Skip-Gram model [17] to train 100-dimensional word embeddings on the corresponding corpus. We set the background word distribution weight $\alpha=0.2$, the number of pseudo documents per class for pre-training $\beta=500$, the size of class-specific vocabulary $\gamma=50$ and the self-training stopping criterion $\delta=0.1$.

We apply our proposed framework on two types of state-ofthe-art text classification neural models: (1) CNN model, whose filter window sizes are 2, 3, 4, 5 with 20 feature maps each. (2) HAN model, which uses a forward GRU with 100 dimension output for both word and sentence encoding. Both the pre-training and the self-training steps are performed using SGD with batch size 256.

The seed information we use as weak supervision for different datasets are described as follows: (1) When the supervision source is label surface name, we directly use the label surface names of all classes; (2) When the supervision source is class-related keywords, we manually choose 3 keywords which do not include the class label name for each class. The selected keywords are shown in Tables 2, 3 and 4, and we evaluate how our model is sensitive to such seed keyword selection in Section 6.6.1; (3) When the supervision source is labeled documents, we randomly sample $c$ documents of each class from the corpus $(c=10$ for The New York Times and AG's News; $c=20$ for Yelp Review) and use them as the given labeled documents. To alleviate the randomness, we repeat the document selection process 10 times and show the performances with average and standard deviation values.

\subsection{Experiment Results}

In this subsection, we report our experimental results and our findings.

6.4.1 Overall Text Classification Performance. In the first set of experiments, we compare the classification performance of our method against all the baseline methods on the three datasets. Both macro-F1 and micro-F1 metrics are used to quantify the performance of different methods. As shown in Tables 5 and 6, our proposed framework achieves the overall best performances among all the baselines on three datasets with different weak supervision sources. Specifically, in almost every case, WESTCLAss-CNN yields the best performance among all methods; WESTCLAssHAN performs slightly worse than WESTCLAss-CNN but still

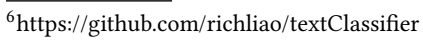


Table 1: Dataset Statistics.

\begin{tabular}{cccc}
\hline Corpus name & Classification type & Class name (Number of documents in the class) & Average document length \\
\hline The New York Times & Topic & Politics (1451), Arts (1043), Business (1429), Science (519), Sports (8639) & 778 \\
AG’s News & Topic & Politics (30000), Sports (30000), Business (30000), Technology (30000) & 45 \\
Yelp Review & Sentiment & Good (19000), Bad (19000) & 155 \\
\hline
\end{tabular}

Table 2: Keyword Lists for The New York Times Dataset.

\begin{tabular}{cc}
\hline Class & Keyword List \\
\hline Politics & \{democracy, religion, liberal \\
Arts & \{music, movie, dance \\
Business & \{investment, economy, industry\} \\
Science & $\{$ scientists, biological, computing\} \\
Sports & $\{$ hockey, tennis, basketball\} \\
\hline
\end{tabular}

Table 3: Keyword Lists for AG's News Dataset.

\begin{tabular}{cc}
\hline Class & Keyword List \\
\hline Politics & \{government, military, war\} \\
Sports & $\{$ basketball, football, athletes\} \\
Business & \{stocks, markets, industries\} \\
Technology & $\{$ computer, telescope, software \\
\hline
\end{tabular}

Table 4: Keyword Lists for Yelp Review Dataset.

\begin{tabular}{cc}
\hline Class & Keyword List \\
\hline Good & \{terrific, great, awesome \\
Bad & \{horrible, disappointing, subpar \\
\hline
\end{tabular}

outperforms other baselines. We discuss the effectiveness of WESTCLAss from the following aspects:

(1) When labeled documents are given as the supervision source, the standard deviation values of WESTCLASS-CNN and WESTCLAss-HAN are smaller than those of CNN and HAN, respectively. This shows that WESTCLASS can effectively reduce the seed sensitivity and improve the robustness of CNN and HAN models.

(2) When the supervision source is label surface name or classrelated keywords, we can see that WESTCLAss-CNN and WESTCLASS-HAN outperform CNN and HAN, respectively. This demonstrates that pre-training with generated pseudo documents results in a better neural model initialization compared to pre-training with documents that are labeled using either IR with tf-idf or Topic Modeling.

(3) WESTCLASS-CNN and WESTCLASS-HAN always outperform NoST-CNN and NoST-HAN, respectively. Note that the only difference between WESTCLASS-CNN/WESTCLASS-HAN and NoST-CNN/NoST-HAN is that the latter two do not include the self-training module. The performance gaps between them thus clearly demonstrate the effectiveness of our self-training module.

6.4.2 Effect of self-training module. In this set of experiments, we conduct more experiments to study the effect of self-training module in WESTCLASS, by investigating the performance of difference models as the number of iterations increases. The results are shown in Figure 2. We can see that the self-training module can effectively improve the model performance after the pre-training step. Also,
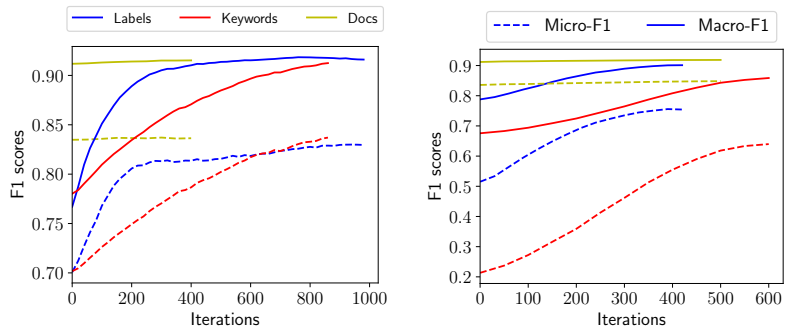

(a) WeSTClass-CNN - New York Times (b) WeSTClass-HAN - New York Times

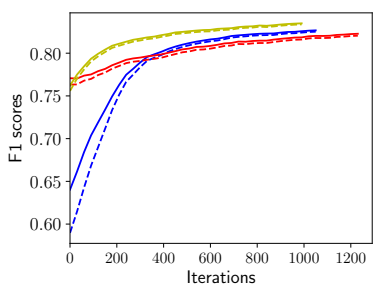

(c) WESTCLASS-CNN - AG's News

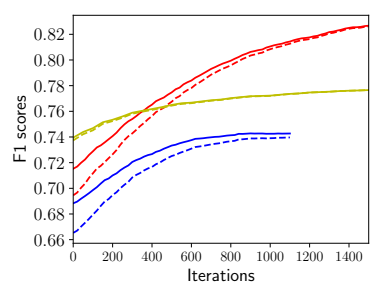

(e) WeSTCLASS-CNN - Yelp Review

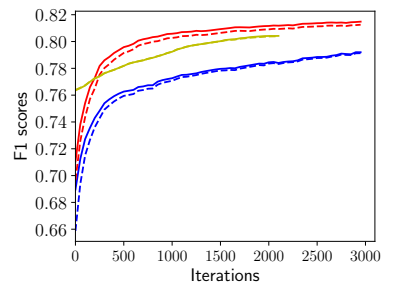

(d) WESTCLASS-HAN - AG's News

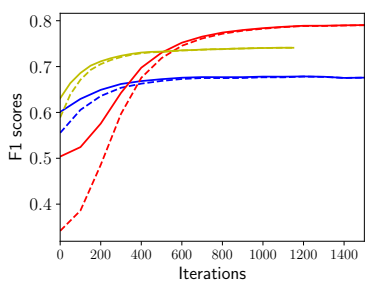

(f) WeSTCLASS-HAN - Yelp Review
Figure 2: Effect of self-training modules on three datasets.

we find that the self-training module generally has the least effect when supervision comes from labeled documents. One possible explanation is that when labeled documents are given, we will use both pseudo documents and provided labeled documents to pretrain the neural models. Such mixture training can often lead to better model initialization, compared to using pseudo documents only. As a result, there is less room for self-training module to make huge improvements.

6.4.3 Effect of the number of labeled documents. When weak supervision signal comes from labeled documents, the setting is similar to semi-supervised learning except that the amount of labeled documents is very limited. In this set of experiments, we vary the number of labeled documents per class and compare the performances of five methods on the AG's News dataset: CNN, HAN, PTE, WESTClass-CNN and WeSTClass-HAN. Again, we run each method 10 times with different sets of labeled documents, and report the average performances with standard deviation (represented as error 
Table 5: Macro-F1 scores for all methods on three datasets. LABELS, KEYWORDS, and DOCS means the type of seed supervision is label surface name, class-related keywords, and labeled documents, respectively.

\begin{tabular}{|c|c|c|c|c|c|c|c|c|c|}
\hline \multirow[t]{2}{*}{ Methods } & \multicolumn{3}{|c|}{ The New York Times } & \multicolumn{3}{|c|}{ AG's News } & \multicolumn{3}{|c|}{ Yelp Review } \\
\hline & LABELS & KEYWORDS & DOCS & LABELS & KEYWORDS & DOCS & LABELS & KEYWORDS & DOCS \\
\hline IR with tf-idf & 0.319 & 0.509 & - & 0.187 & 0.258 & - & 0.533 & 0.638 & - \\
\hline Topic Model & 0.301 & 0.253 & - & 0.496 & 0.723 & - & 0.333 & 0.333 & - \\
\hline Dataless & 0.484 & - & - & 0.688 & - & - & 0.337 & - & - \\
\hline UNEC & 0.690 & - & - & 0.659 & - & - & 0.602 & - & - \\
\hline PTE & - & - & $0.834(0.024)$ & - & - & $0.542(0.029)$ & - & - & $0.658(0.042)$ \\
\hline HAN & 0.348 & 0.534 & $0.740(0.059)$ & 0.498 & 0.621 & $0.731(0.029)$ & 0.519 & 0.631 & $0.686(0.046)$ \\
\hline $\mathrm{CNN}$ & 0.338 & 0.632 & $0.702(0.059)$ & 0.758 & 0.770 & $0.766(0.035)$ & 0.523 & 0.633 & $0.634(0.096)$ \\
\hline NoST-HAN & 0.515 & 0.213 & $0.823(0.035)$ & 0.590 & 0.727 & $0.745(0.038)$ & 0.731 & 0.338 & $0.682(0.090)$ \\
\hline NoST-CNN & 0.701 & 0.702 & $0.833(0.013)$ & 0.534 & 0.759 & $0.759(0.032)$ & 0.639 & 0.740 & $0.717(0.058)$ \\
\hline WESTCLASS-HAN & 0.754 & 0.640 & $0.832(0.028)$ & 0.816 & 0.820 & $0.782(0.028)$ & 0.769 & 0.736 & $0.729(0.040)$ \\
\hline WESTCLASS-CNN & 0.830 & 0.837 & $0.835(0.010)$ & 0.822 & 0.821 & $0.839(0.007)$ & 0.735 & 0.816 & $0.775(0.037)$ \\
\hline
\end{tabular}

Table 6: Micro-F1 scores for all methods on three datasets. LABELS, KEYWORDS, and DOCS means the type of seed supervision is label surface name, class-related keywords, and labeled documents, respectively.

\begin{tabular}{|c|c|c|c|c|c|c|c|c|c|}
\hline \multirow[t]{2}{*}{ Methods } & \multicolumn{3}{|c|}{ The New York Times } & \multicolumn{3}{|c|}{ AG's News } & \multicolumn{3}{|c|}{ Yelp Review } \\
\hline & LABELS & KEYWORDS & DOCS & LABELS & KEYWORDS & DOCS & LABELS & KEYWORDS & DOCS \\
\hline IR with tf-idf & 0.240 & 0.346 & - & 0.292 & 0.333 & - & 0.548 & 0.652 & - \\
\hline Topic Model & 0.666 & 0.623 & - & 0.584 & 0.735 & - & 0.500 & 0.500 & - \\
\hline Dataless & 0.710 & - & - & 0.699 & - & - & 0.500 & - & - \\
\hline UNEC & 0.810 & - & - & 0.668 & - & - & 0.603 & - & - \\
\hline PTE & - & - & $0.906(0.020)$ & - & - & $0.544(0.031)$ & - & - & $0.674(0.029)$ \\
\hline HAN & 0.251 & 0.595 & $0.849(0.038)$ & 0.500 & 0.619 & $0.733(0.029)$ & 0.530 & 0.643 & $0.690(0.042)$ \\
\hline $\mathrm{CNN}$ & 0.246 & 0.620 & $0.798(0.085)$ & 0.759 & 0.771 & $0.769(0.034)$ & 0.534 & 0.646 & $0.662(0.062)$ \\
\hline NoST-HAN & 0.788 & 0.676 & $0.906(0.021)$ & 0.619 & 0.736 & $0.747(0.037)$ & 0.740 & 0.502 & $0.698(0.066)$ \\
\hline NoST-CNN & 0.767 & 0.780 & $0.908(0.013)$ & 0.553 & 0.766 & $0.765(0.031)$ & 0.671 & 0.750 & $0.725(0.050)$ \\
\hline WESTCLASS-HAN & 0.901 & 0.859 & $0.908(0.019)$ & 0.816 & 0.822 & $0.782(0.028)$ & 0.771 & 0.737 & $0.729(0.040)$ \\
\hline WESTCLASS-CNN & 0.916 & 0.912 & $0.911(0.007)$ & 0.823 & 0.823 & $0.841(0.007)$ & 0.741 & 0.816 & $0.776(0.037)$ \\
\hline
\end{tabular}

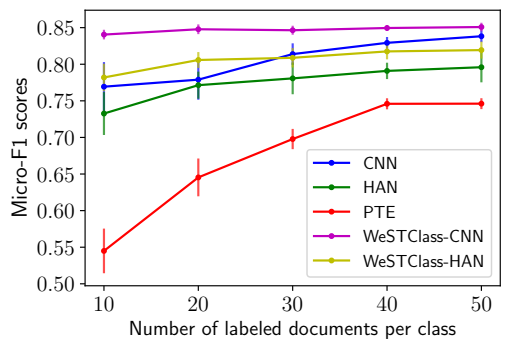

Figure 3: The performances of different methods on AG's News dataset when the number of labeled documents varies.

bars) in Figure 3. We can see that when the amount of labeled documents is relatively large, the performances of the five methods are comparable. However, when fewer labeled documents are provided, PTE, CNN and HAN not only exhibit obvious performance drop, but also become very sensitive to the seed documents. Nevertheless, WESTCLAss-based models, especially WESTCLASs-CNN, yield stable performance with varying amount of labeled documents. This phenomenon shows that our method can more effectively take advantage of the limited amount of seed information to achieve better performance.

\subsection{Parameter Study}

In this section, we study the effects of different hyperparameter settings on the performance of WESTCLAss with CNN and HAN models, including (1) background word distribution weight $\alpha$, (2) number of generated pseudo documents $\beta$ for pre-training and (3) keyword vocabulary size $\gamma$ used in equation (1) where $\gamma=\left|V_{d_{i}}\right|$. When studying the effect of one parameter, the other parameters are set to their default values as described in Section 6.3. We conduct all the parameter studies on the AG's News dataset.

6.5.1 Background Word Distribution Weight. The background word distribution weight $\alpha$ is used in both the language model for pseudo documents generation and pseudo-labels computation. When $\alpha$ becomes smaller, the generated pseudo documents contain more topic-related words and fewer background words, and the pseudolabels become similar to one-hot encodings. We vary $\alpha$ from 0 to 1 with interval equal to 0.1 . The effect of $\alpha$ is shown in Figure 4. Overall, different $\alpha$ values result in comparable performance, except when $\alpha$ is close to 1, pseudo documents and pseudo-labels become uninformative: pseudo documents are generated directly from background word distribution without any topic-related information, and pseudo-labels are uniform distributions. We notice that when $\alpha=1$, labeled documents as supervision source results in much 


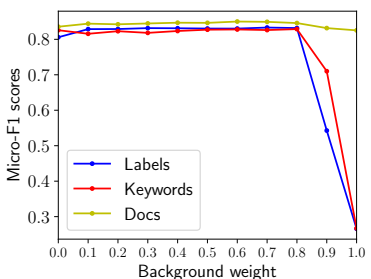

(a) WeSTCLASS-CNN

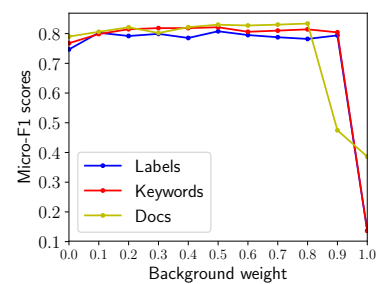

(b) WESTCLASS-HAN
Figure 4: Effect of background word distribution weight $\alpha$ on AG's News dataset.

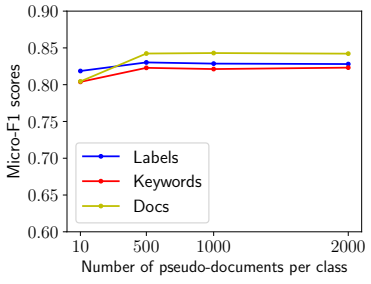

(a) WeSTClass-CNN

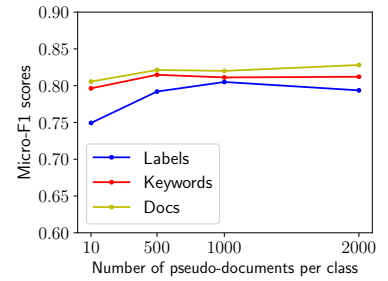

(b) WeSTCLASS-HAN
Figure 5: Effect of pseudo documents amount per class $\beta$ for pre-training on AG's News dataset.

better performance than label surface name and class-related keywords. This is because pre-training with labeled documents is performed using both pseudo documents and labeled documents, and the provided labeled documents are still informative. When $\alpha$ is close to 0 , the performance is slightly worse than other settings, because pseudo documents only contain topic-related keywords and pseudo-labels are one-hot encodings, which can easily lead to model overfitting to pseudo documents and behaving worse on real documents classification.

6.5.2 Number of pseudo documents for pre-training. The effect of pseudo documents amount $\beta$ is shown in Figure 5 . We have the following findings from Figure 5: On the one hand, if the amount of generated pseudo documents is too small, the information carried in pseudo documents will be insufficient to pre-train a good model. On the other hand, generating too many pseudo documents will make the pre-training process unnecessarily long. Generating 500 to 1000 pseudo documents of each class for pre-training will strike a good balance between pre-training time and model performance.

6.5.3 Size of Keyword Vocabulary. Recall the pseudo document generation process in Section 4.2, after sampling a document vector $\boldsymbol{d}_{i}$, we will first construct a keyword vocabulary $V_{d_{i}}$ that contains the top- $\gamma$ words with most similar word embedding with $\boldsymbol{d}_{i}$. The size of the keyword vocabulary $\gamma$ controls the number of unique words that appear frequently in the generated pseudo documents. If $\gamma$ is too small, only a few topical keywords will appear frequently in pseudo documents, which will reduce the generalization ability of the pre-trained model. As shown in Figure 6, $\gamma$ can be safely set within a relatively wide range from 50 to 500 in practice.

\subsection{Case Study}

In this subsection, we perform a set of case studies to further understand the properties of our proposed method.

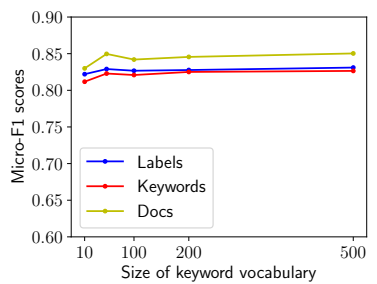

(a) WeSTClass-CNN

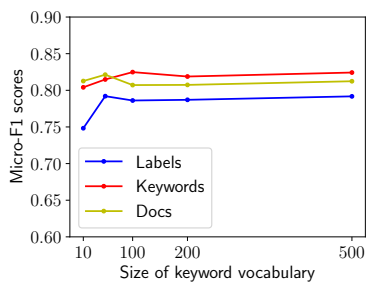

(b) WeSTClass-HAN
Figure 6: Effect of keyword vocabulary size $\gamma$ on AG's News dataset.

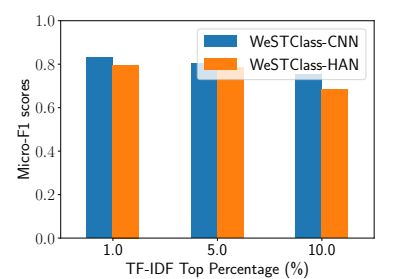

(a)

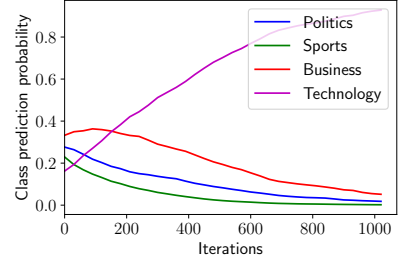

(b)
Figure 7: (a) Performances on AG's News dataset under different sets of seed keywords. (b) Class prediction probability during self-training procedure for a sample document.

6.6.1 Choice of Seed Keywords. In the first set of case studies, we are interested in how sensitive our model is to the selection of seed keywords. In Section 6.3, we manually select class-related keywords, which could be subjective. Here we explore the sensitivity of WESTCLAss-CNN and WESTCLAss-HAN to different sets of seed keywords. For each class $j$ of AG's News dataset, we first collect all documents belonging to class $j$, and then compute the tf-idf weighting of each word in each document of class $j$. We sort each word's average tf-idf weighting in these documents from high to low. Finally we form the seed keyword lists by finding words that rank at top $1 \%$ (most relevant), $5 \%$ and $10 \%$ based on the average tf-idf value. The keywords of each class at these percentages are shown in Table 7; the performances of WESTCLAss-CNN and WESTCLAss-HAN are shown in Figure 7(a). At top 5\% and $10 \%$ of the average tf-idf weighting, although some keywords are already slightly irrelevant to their corresponding class semantic, WESTCLASS-CNN and WESTCLASS-HAN still perform reasonably well, which shows the robustness of our proposed framework to different sets of seed keywords.

6.6.2 Self-training Corrects Misclassification. In the second set of case studies, we are interested in how the self-training module behaves to improve the performance of our model. Figure 7(b) shows WESTCLASs-CNN's prediction with label surface name as supervision source on a sample document from AG's News dataset: The national competition regulator has elected not to oppose Telstra's $3 G$ radio access network sharing arrangement with rival telco Hutchison. We notice that this document is initially misclassified after the pre-training procedure, but it is then corrected by the subsequent self-training step. This example shows that neural models have the ability of self-correcting by learning from its high-confidence predictions with appropriate pre-training initialization. 
Table 7: Keyword Lists at Top Percentages of Average Tf-idf.

\begin{tabular}{cccc}
\hline Class & $1 \%$ & $5 \%$ & $10 \%$ \\
\hline Politics & \{government, president, minister\} & \{mediators, criminals, socialist\} & \{suspending, minor, lawsuits\} \\
Sports & \{game, season, team\} & \{judges, folks, champagne & \{challenging, youngsters, stretches\} \\
Business & \{profit, company, sales\} & \{refunds, organizations, trader\} & $\{$ winemaker, skilling, manufactured\} \\
Technology & $\{$ internet, web, microsoft\} & $\{$ biologists, virtually, programme & \{demos, microscopic, journals\} \\
\hline
\end{tabular}

\section{DISCUSSIONS AND CONCLUSIONS}

We have proposed a weakly-supervised text classification method built upon neural classifiers. With (1) a pseudo document generator for generating pseudo training data and (2) a self-training module that bootstraps on real unlabled data for model refining, our method effectively addresses the key bottleneck for existing neural text classifiers-the lack of labeled training data. Our method is not only flexible in incorporating difference sources of weak supervision (class label surface names, class-related keywords, and labeled documents), but also generic enough to support different neural models (CNN and RNN). Our experimental results have shown that our method outperforms baseline methods significantly, and it is quite robust to different settings of hyperparameters and different types of user-provided seed information.

An interesting finding based on the experiments in Section 6 is that different types of weak supervision are all highly helpful for the good performances of neural models. In the future, it is interesting to study how to effectively integrate different types of seed information to further boost the performance of our method.

\section{ACKNOWLEDGEMENTS}

This research is sponsored in part by U.S. Army Research Lab. under Cooperative Agreement No. W911NF-09-2-0053 (NSCTA), DARPA under Agreement No. W911NF-17-C-0099, National Science Foundation IIS 16-18481, IIS 17-04532, and IIS-17-41317, DTRA HDTRA11810026, and grant 1U54GM114838 awarded by NIGMS through funds provided by the trans-NIH Big Data to Knowledge (BD2K) initiative (www.bd2k.nih.gov). We thank anonymous reviewers for valuable and insightful feedback.

\section{REFERENCES}

[1] Dzmitry Bahdanau, Kyunghyun Cho, and Yoshua Bengio. 2014. Neural Machine Translation by Jointly Learning to Align and Translate. CoRR abs/1409.0473 (2014).

[2] Arindam Banerjee, Inderjit S. Dhillon, Joydeep Ghosh, and Suvrit Sra. 2005 Clustering on the Unit Hypersphere using von Mises-Fisher Distributions. Journal of Machine Learning Research (2005).

[3] Kayhan Batmanghelich, Ardavan Saeedi, Karthik Narasimhan, and Samuel Gershman. 2016. Nonparametric Spherical Topic Modeling with Word Embeddings. In $A C L$.

[4] David M. Blei, Andrew Y. Ng, and Michael I. Jordan. 2003. Latent Dirichlet Allocation. In NIPS.

[5] Ming-Wei Chang, Lev-Arie Ratinov, Dan Roth, and Vivek Srikumar. 2008. Importance of Semantic Representation: Dataless Classification. In AAAI.

[6] Xingyuan Chen, Yunqing Xia, Peng Jin, and John A. Carroll. 2015. Dataless Text Classification with Descriptive LDA. In AAAI.

[7] Ronald Fisher. 1953. Dispersion on a sphere. Proceedings of the Royal Society of London. Series A. Mathematical and Physical Sciences (1953).

[8] Evgeniy Gabrilovich and Shaul Markovitch. 2007. Computing Semantic Relatedness Using Wikipedia-based Explicit Semantic Analysis. In IfCAI.

[9] Kuzman Ganchev, João GraÃğa, Jennifer Gillenwater, and Ben Taskar. 2010. Posterior Regularization for Structured Latent Variable Models. Fournal of Machine Learning Research (2010).

[10] Siddharth Gopal and Yiming Yang. 2014. Von Mises-Fisher Clustering Models. In ICML.
[11] Rie Johnson and Tong Zhang. 2015. Effective Use of Word Order for Text Categorization with Convolutional Neural Networks. In HLT-NAACL.

[12] Yoon Kim. 2014. Convolutional Neural Networks for Sentence Classification. In EMNLP.

[13] Omer Levy, Yoav Goldberg, and Ido Dagan. 2015. Improving Distributional Similarity with Lessons Learned from Word Embeddings. TACL (2015).

[14] Chenliang Li, Jian Xing, Aixin Sun, and Zongyang Ma. 2016. Effective Document Labeling with Very Few Seed Words: A Topic Model Approach. In CIKM.

[15] Keqian Li, Hanwen Zha, Yu Su, and Xifeng Yan. 2018. Unsupervised Neural Categorization for Scientific Publications. In SDM.

[16] Yue Lu and Chengxiang Zhai. 2008. Opinion integration through semi-supervised topic modeling. In $W W W$.

[17] Tomas Mikolov, Ilya Sutskever, Kai Chen, Gregory S. Corrado, and Jeffrey Dean. 2013. Distributed Representations of Words and Phrases and their Compositionality. In NIPS.

[18] Takeru Miyato, Andrew M. Dai, and Ian Goodfellow. 2016. Adversarial Training Methods for Semi-Supervised Text Classification.

[19] Kamal Nigam and Rayid Ghani. 2000. Analyzing the Effectiveness and Applicability of Co-training. In CIKM.

[20] Avital Oliver, Augustus Odena, Colin Raffel, Ekin D. Cubuk, and Ian J. Goodfellow. 2018. Realistic Evaluation of Semi-Supervised Learning Algorithms.

[21] Chuck Rosenberg, Martial Hebert, and Henry Schneiderman. 2005. SemiSupervised Self-Training of Object Detection Models. In WACV/MOTION.

[22] Richard Socher, Eric H. Huang, Jeffrey Pennington, Andrew Y. Ng, and Christopher D. Manning. 2011. Dynamic Pooling and Unfolding Recursive Autoencoders for Paraphrase Detection. In NIPS.

[23] Richard Socher, Jeffrey Pennington, Eric H. Huang, Andrew Y. Ng, and Christopher D. Manning. 2011. Semi-Supervised Recursive Autoencoders for Predicting Sentiment Distributions. In EMNLP.

[24] Yangqiu Song and Dan Roth. 2014. On Dataless Hierarchical Text Classification. In $A A A I$.

[25] Suvrit Sra. 2016. Directional statistics in machine learning: a brief review. arXiv preprint arXiv:1605.00316 (2016).

[26] Suvrit Sra and Sharon K Sra. 2011. A short note on parameter approximation for von Mises-Fisher distributions: and a fast implementation of Is(x).

[27] Duyu Tang, Bing Qin, and Ting Liu. 2015. Document Modeling with Gated Recurrent Neural Network for Sentiment Classification. In EMNLP.

[28] Jian Tang, Meng Qu, and Qiaozhu Mei. 2015. PTE: Predictive Text Embedding through Large-scale Heterogeneous Text Networks. In KDD.

[29] Gilad Tsur, Yuval Pinter, Idan Szpektor, and David Carmel. 2016. Identifying Web Queries with Question Intent. In $W W W$.

[30] Junyuan Xie, Ross B. Girshick, and Ali Farhadi. 2016. Unsupervised Deep Embedding for Clustering Analysis. In ICML.

[31] Weidi Xu, Haoze Sun, Chao Deng, and Ying Tan. 2017. Variational Autoencoder for Semi-Supervised Text Classification. In AAAI.

[32] Zichao Yang, Diyi Yang, Chris Dyer, Xiaodong He, Alexander J. Smola, and Eduard H. Hovy. 2016. Hierarchical Attention Networks for Document Classification. In HLT-NAACL.

[33] Zichao Yang, Diyi Yang, Chris Dyer, Xiaodong He, Alexander J. Smola, and Eduard H. Hovy. 2016. Hierarchical Attention Networks for Document Classification. In HLT-NAACL.

[34] Chao Zhang, Liyuan Liu, Dongming Lei, Quan Yuan, Honglei Zhuang, Tim Hanratty, and Jiawei Han. 2017. TrioVecEvent: Embedding-Based Online Local Event Detection in Geo-Tagged Tweet Streams. In KDD. 595-604.

[35] Xiang Zhang and Yann LeCun. 2015. Text Understanding from Scratch. CoRR abs/1502.01710 (2015).

[36] Xiang Zhang, Junbo Jake Zhao, and Yann LeCun. 2015. Character-level Convolutional Networks for Text Classification. In NIPS 